\title{
Role of Sonography in Clinically Occult Femoral Hernias
}

\author{
David W. Brandel, MD, Gandikota Girish, MBBS, Catherine J. Brandon, MD, Qian Dong, MD, Corrie \\ Yablon, MD, David A. Jamadar, MBBS
}

Received February 25, 2015, from the University of Michigan Health System, Ann Arbor, Michigan USA. Revision requested April 6, 2015. Revised manuscript accepted for publication May 6, 2015.

Address correspondence to Gandikota Girish, MBBS, University of Michigan Health System, Taubman Center, Floor 2, Reception A, 1500 E. Medical Center Dr, SPC 5326, Ann Arbor, MI 48109 USA.

E-mail:ggirish@med.umich.edu
Objectives - The purpose of this article is to evaluate the diagnostic accuracy of sonography in clinically occult femoral hernias and to describe our sonographic technique.

Methods - The clinical and imaging data for 93 outpatients referred by general surgeons, all of whom underwent sonographic evaluation and surgery, were reviewed retrospectively. Of these, 55 patients who underwent surgical exploration for groin hernias within 3 months of sonography and met all inclusion criteria were included in the study. The sonographic technique involves using the pubic tubercle as an osseous landmark to identify and appropriately visualize the femoral canal. The Valsalva maneuver is then used to differentiate the movement of normal fat (a potential pitfall) from true herniation in the femoral canal. Surgical findings were used as the reference standard by which sonographic results were judged. Two-by-two contingency tables were used to calculate the sensitivity, specificity, positive predictive value, and negative predictive value.

Results - In these 55 patients, surgery revealed 15 femoral hernias. Eight femoral hernias occurred in women, and 7 occurred in men. For diagnosing femoral hernias, sonography demonstrated sensitivity of $80 \%$, specificity of $88 \%$, a positive predictive value of $71 \%$, and a negative predictive value of $92 \%$. True-positive cases of femoral hernias have a sonographic appearance of a hypoechoic sac with speckled internal echoes. When examining during the Valsalva maneuver, a femoral hernia passes deep to the inguinal ligament, expands the femoral canal, displacing the normal canal fat, and effaces the femoral vein.

Conclusions - Sonography can exclude femoral hernias with high confidence in light of its exceptional negative predictive value. With attention to technique and imaging criteria, the diagnostic accuracy of sonography can be enhanced.

Key Words - femoral canal; femoral hernia; hernia diagnosis; hernia evaluation; occult hernia; superficial structures

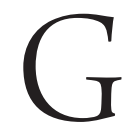
roin pain is a common presenting symptom in adult patients, and herniorraphy is the most common surgical procedure in the United States, with more than 800,000 operations performed annually. ${ }^{1}$ As there are multiple etiologies for this presentation, the differential diagnosis of groin pain depends first and foremost on the clinical history and physical examination. With regard to hernias, classic findings have been well described in the literature and are highly reliable when the combination of a palpable lump and transmission of a cough impulse are present. ${ }^{2}$ 
However, physical examination may be unremarkable in some patients with small hernias and difficult to interpret in others. When the diagnosis of a femoral hernia is clinically equivocal, sonography is a highly accessible, inexpensive, dynamic, and noninvasive method of obtaining additional information. The utility of sonography is well established in the diagnosis of direct and indirect inguinal hernias, ${ }^{3-8}$ although its performance with regard to occult femoral hernias is not well documented.

A femoral hernia is much more likely to be nonpalpable, or "occult," than the more common inguinal hernia and is more difficult to diagnose clinically. ${ }^{9}$ Femoral hernias occur in the short femoral canal found along the medial side of the femoral vein. The pectineus muscle forms the floor of the canal; the inguinal ligament is the cranial roof; and the lacunar ligament acts as the medial wall (Figure 1A). There are several variant pathways for femoral hernia formation (Figure 1B). Femoral hernias are more likely to become incarcerated because of the relatively rigid and narrow neck. ${ }^{10,11}$ The combination of these features contributes to increased health care costs and patient morbidity, since up to half of all initially diagnosed femoral hernias require immediate hospitalization and surgical repair., ${ }^{9,11,12}$ Emergent repair is much more likely to be associated with an adverse surgical outcome and carries a 7 -fold higher mortality risk compared to elective hernia repair, which increases to a 20 -fold mortality risk if bowel resection is undertaken. ${ }^{9,12}$ Despite these poor clinical outcomes, little data are available regarding the utility of sonography in the diagnosis of occult femoral hernias. We review the anatomy and sonographic technique for evaluation of the femoral canal and present our results for diagnosis of clinically occult femoral hernias.

\section{Materials and Methods}

\section{Patient Population}

Between January 2005 and June 2012, general surgeons referred 271 patients presenting to outpatient clinics with groin pain for sonographic evaluation of clinically equivocal hernias at a large teaching hospital. Sonographic examinations of the inguinal and femoral region were conducted on the side of the patient's symptoms. From the electronic medical record, clinical data regarding these patients were reviewed. Patients were included in this study if they underwent a sonographic examination and a subsequent surgical exploration within 3 months after the sonogram. Seventy-one patients met these criteria. A single patient was excluded because of a non-hernia diagnosis at surgery (reactive lymph nodes). Fifteen patients who had undergone prior hernia repair with or without indwelling mesh were also excluded to reduce potential confounding variables. These exclusions yielded a study population of 55 patients. Fifteen patients had a specific diagnosis of a femoral hernia at surgery, our reference standard. Appropriate Institutional Review Board approval was obtained before the onset of this study.

\section{Sonographic Technique}

At our institution, the inguinal and femoral regions are evaluated with a linear probe, given their relatively superficial nature. A $12-\mathrm{MHz}$ probe is most commonly selected, as it provides excellent sonographic resolution, although a lower-frequency probe may be needed in patients with a larger body habitus. The femoral canal is examined in both the axial (transverse) and sagittal (longitudinal) planes with and without techniques to increase intra-abdominal pressure (ie, Valsalva maneuver). In the axial plane, the transducer is held with its medial edge on the pubic tubercle, which is the optimal location for identifying the femoral canal's axial plane (Figure 1A). In our experience, from this initial transducer location, scanning $1 \mathrm{~cm}$ cranially and $1 \mathrm{~cm}$ caudally adequately covers the femoral canal in most patients and should include most of the anatomic variants of hernia formation (Figure 1B). Identifying the long axis of the femoral canal is accomplished by placing the transducer parallel to the long axis of the external iliac artery/ common femoral artery where it passes over the pectineus muscle at the pelvic brim. By moving the transducer in this plane medially, the long axis of the femoral vein will be visualized, and further movement medially will place the transducer over the femoral canal when the femoral vein is just out of the field of view. In this plane, the femoral canal starts deep to the inguinal canal at the caudal extent of the spermatic cord/round ligament, which may be seen in cross section. In our experience, pain or tenderness is a useful feature in guiding the operator toward the location of a potential hernia but is not specific to an occult femoral hernia, the diagnosis of which is based on imaging features.

\section{Data Analysis}

A femoral hernia was considered present only if explicitly mentioned in the surgical note. We reviewed the pertinent imaging in all of our surgically confirmed positive, falsenegative, and false-positive cases. Additionally, images from 20 surgically confirmed true-negative studies (no femoral hernia identified during sonography or surgery) were examined. Two-by-two contingency tables were used to calculate the sensitivity, specificity, positive predictive value, and negative predictive value. 


\section{Results}

The total study population included 41 male patients (75\%) and 14 female patients (25\%). Of the 15 surgically confirmed femoral hernias, 9 were right sided and 6 were left sided. Eight of these patients were female, and 7 were male. Although no significant sex predilection was observed in absolute numbers, fewer female patients were referred for sonography. Taking this factor into consideration, a higher percentage of female patients were surgically diagnosed with femoral hernias (8 of $14[57 \%]$ ) than male patients (7 of $41[17 \%]$ ). Women found to have femoral hernias at surgery varied in age from 27 to 86 years, with a median age of 45 years and a mean age of 50 years. Men with surgical diagnoses of femoral hernias ranged from 17 to 80 years, with a median age of 33 years and a mean age of 37 years. All of the femoral hernias observed contained only preperitoneal fat (no bowel), and 3 of these hernias were found to be incarcerated (nonreducible) at exploration, although none showed evidence of vascular compromise (strangulation). Twelve of 15 patients with positive findings for femoral hernias at surgery were prospectively identified by sonography, yielding sensitivity of $80 \%$. Additionally, sonography correctly diagnosed the hernia sac as containing only preperitoneal fat in all 12 cases, and the hernias were nonreducible in all 3 surgically confirmed incarcerated cases ( 1 male and 2 female).

Of the 55 patients who underwent surgical exploration, 40 had a surgically normal femoral canal. Six of these patients were female, and 35 were male. Of these 40 patients, 34 were described prospectively as having negative findings for femoral hernias by sonography, yielding specificity of $88 \%$. A positive sonographic finding correctly predicted a femoral hernia at surgery in 12 of 17 cases, yielding a positive predictive value of $71 \%$. Negative sonographic findings correctly predicted the absence of a femoral hernia in 35 of 38 cases, yielding a negative predictive value of $92 \%$.

\section{Discussion}

In 1981, Deitch and Soncrant ${ }^{13}$ first described the use of sonography to assess nonpalpable femoral hernias and prospectively identified a "space-occupying mass medial to the femoral vein" in 4 patients, all of whom had a surgically confirmed femoral hernia. These authors also emphasized the potential advantages of sonography to confirm this diagnosis in clinically equivocal scenarios, given the high incidence of incarceration and strangulation, and rec-

Figure 1. A, Illustration of a dissected right femoral region from the anterior view shows the fat in the femoral canal (F) adjacent to the femoral vein and also shows anatomic relationships with the inguinal ligament (arrows), lacunar ligament (L), iliacus muscle (I), psoas tendon ( $T$ ), and pectineus muscle (P), which lies on the superior pubic ramus (SP). B, Illustration of a dissected right femoral region shows possible variations of hernias occurring in the femoral region. By far the most common is within the femoral canal $(C)$; other types of femoral hernias include lateral $(L)$, prevascular $(P)$, medial (M), and retrovascular (R). SIR indicates superficial inguinal ring.
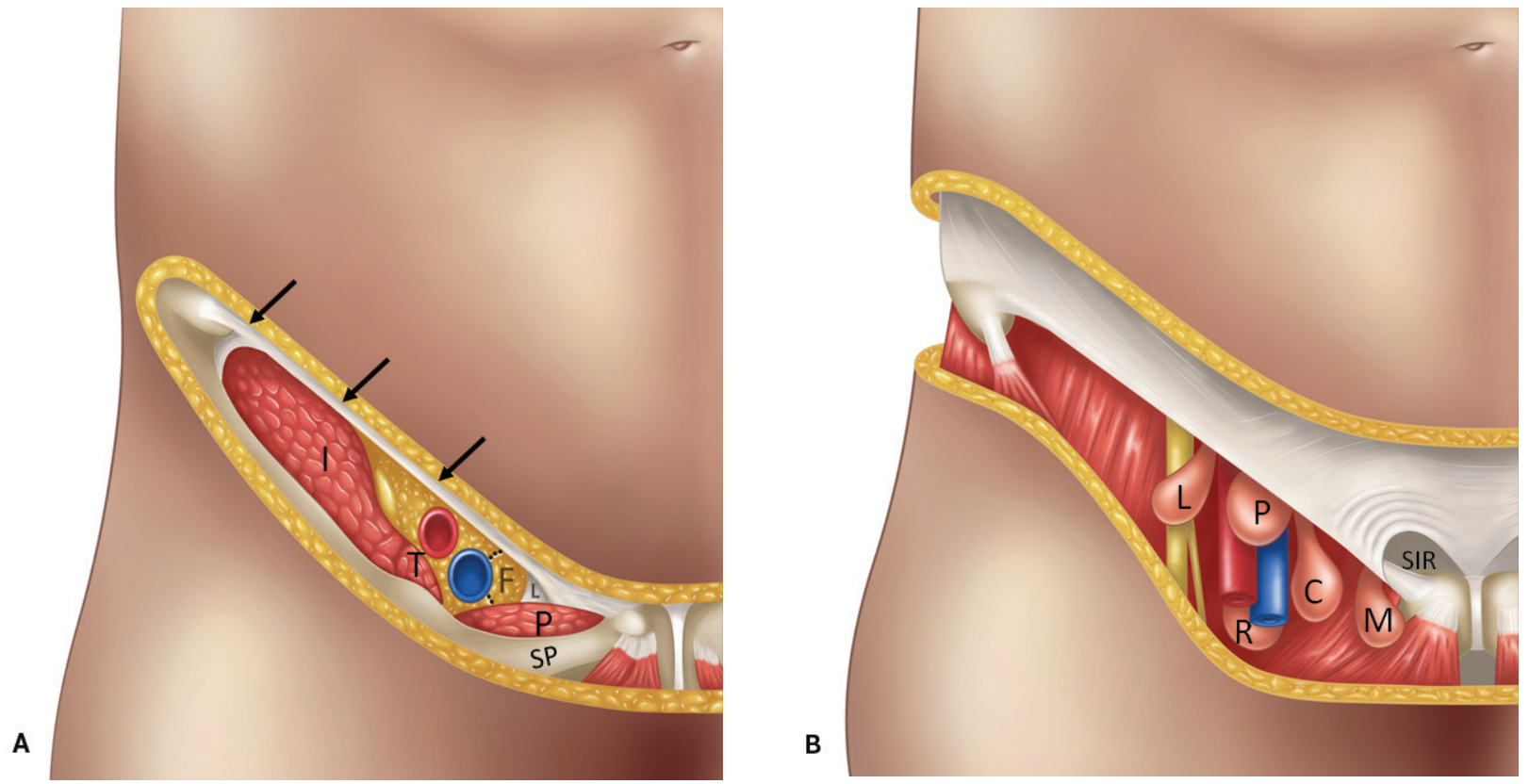
ommended expanding the role of sonography in this context, stating that "liberal use may help in decreasing the unacceptably high morbidity and mortality of this clinically treacherous disease."

Sonographic technology has continued to improve since this index study, and several articles studying the use of sonography for diagnosis of occult inguinal hernias are now available. ${ }^{3-6,14,15}$ Notably, a 2013 meta-analysis found that when compared to computed tomography, sonography showed higher sensitivity ( 0.86 versus 0.79$)$ and specificity ( 0.77 versus 0.69 ) for detection of occult inguinal hernias. ${ }^{15}$ Data regarding the diagnosis of femoral hernias are scarcer, likely in part because of the relatively low prevalence of this hernia subtype. To the best of our knowledge, Depasquale et al, ${ }^{16}$ in 2009, provided the only Englishlanguage article that has specifically addressed the use of sonography for occult femoral hernias since the publication by Dietch and Soncrant ${ }^{13}$ in 1981 . That study retrospectively reviewed clinical data and 243 sonographic evaluations of 243 patients. Ninety-four patients were considered to have positive findings for hernias on sonography, 22 of whom were given a diagnosis of a femoral hernia. ${ }^{16}$ However, of the 94 patients with positive sonographic findings, 32 did not undergo surgery, precluding a calculation of sensitivity and specificity. With regard to femoral hernias, the authors did describe 1 false-negative case, which was prospectively interpreted as an inguinal hernia, and 2 false-positive cases, which were determined to be inguinal hernias at sonography. The fraction of the 22 patients with a sonographic diagnosis of a femoral hernia who underwent subsequent surgical examination was not specified.

In our study, retrospective evaluation of the images of the true-positive against the true-negative cases allowed us to make some important observations. In patients without femoral hernias, normal fat can show a small degree of motion within the femoral canal. Indeed, in their description of technique, Depasquale et $\mathrm{al}^{16}$ stated that an "element of protrusion of pre-peritoneal fat into the femoral canal during Valsalva is common due to natural variability in tissue elasticity and anatomical configuration." In all of our true-negative cases (20 of 20) examined in the axial plane, this preperitoneal fat is echogenic, and whereas it may be seen to abut the femoral vein, it does not truly deform the shape of the vein (Figure 2 and Video 1). In contrast, nearly all of our true-positive cases (14 of 15) showed the following characteristics: (1) the hernia appears as a heterogeneous mass with speckled internal echoes that is hypoechoic to and displaces the normal preperitoneal fat during dynamic maneuvers (Figure 3B and Video 2); (2) during the Valsalva maneuver, there is a routine increase in the normal caliber of the femoral canal, and if there is a hernia, the hernia sac bulges, effacing the normal rounded medial contour of the adjacent femoral vein (Figure 3, A and $\mathrm{B}$, and Video 2); and (3) movement of the femoral hernia sac is from proximal to distal in the femoral canal, paralleling the femoral vessels (Figure 3C and Video 3). These imaging characteristics are summarized in Table 1. Our single exception was a prevascular variant, which showed features unique to its position outside the femoral canal, with no demonstrable effect on the femoral vein (Figure 4).

Using the above characteristics as proposed criteria for sonographic evaluation, our misdiagnosed cases were reviewed by 2 senior radiologists with greater than 30 years of cumulative experience to identify potential sources of error. Regarding 2 of our 3 false-negative cases (no femoral hernia identified on sonography but reported at surgery), consensus review determined that the images did in fact support a femoral hernia, which was misinterpreted prospectively. In both of these cases, the femoral canal was scanned too cranially in the axial plane, and although no hernia was seen in this orientation, the hernia was present on the longitudinal views but was missed or ignored. Our third-false negative case still lacked sufficient diagnostic criteria for a femoral hernia, perhaps the result of submaximal exertion producing little movement with the Valsalva maneuver, a potential pitfall (Videos 4 and 5). Additionally, on review of our false-positive cases, all (5 of 5) showed some movement in the femoral canal during the Valsalva maneuver, and the importance of this movement may have been overestimated during the original interpretation. In all of these 5 cases, the minimal femoral canal movement noted during the Valsalva maneuver did not cause any femoral vein effacement.

In our practice, sonographic assessment for an occult femoral hernia is typically performed in an outpatient setting. This factor may explain why none of our patients had emergent findings, and only 3 of 15 (20\%) showed nonreducibility during dynamic maneuvers. However, we do know that all 3 of our surgically confirmed cases of incarceration, including an anatomic variant, were prospectively diagnosed with sonography (Figure 4), which provisionally suggests that sonography is a useful tool for predicting this clinically important finding. In all 3 confirmed cases of incarceration, the hernia sac was not fully reducible during dynamic maneuvers, and free fluid was seen within the femoral canal distal to the sac (Figure 4). We propose that the combination of a nonreducible hernia and free fluid in the femoral canal is highly suggestive of an incarcerated 
femoral hernia. Indeed, the sonographic finding of free fluid within a hernia sac containing fat, but no bowel, has been shown to correlate well with incarceration at surgery, showing sensitivity of $100 \%$ and specificity of $96 \%{ }^{17}$

Case reports of sonographic diagnosis of occult femoral hernias leading to bowel obstruction exist, ${ }^{18,19}$ although reproducibility and the ultimate utility of this modality in more acute settings remain to be seen. Given that our target population included nonemergent cases of femoral hernias, most of which only manifested themselves with increased abdominal pressure and spontaneously reduced at rest, applying transducer pressure to attempt hernia reduction was not part of our protocol. This technique has been used by surgeons and emergency physi-
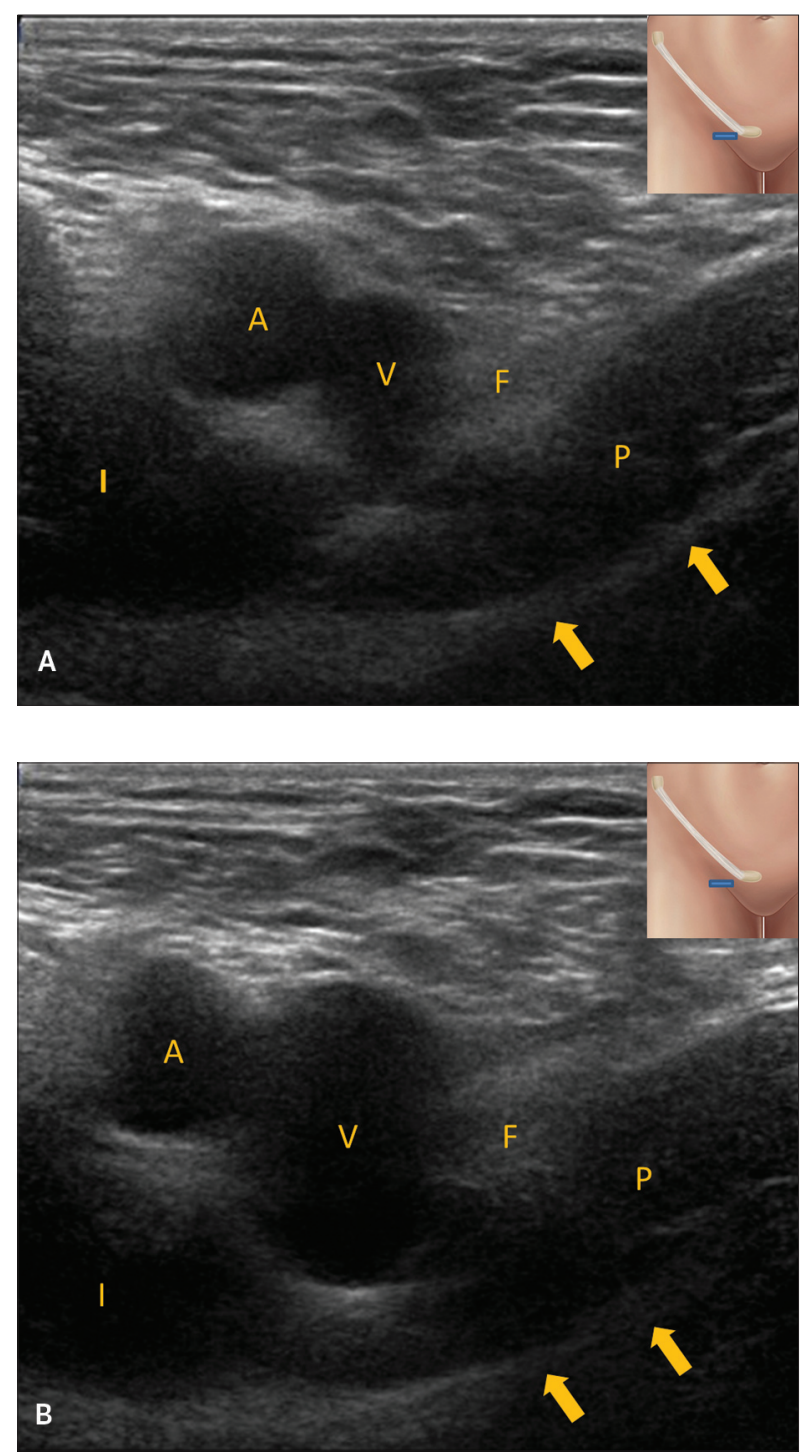

cians with regard to incarcerated inguinal hernias and, if successful may eliminate the need for an emergent surgical procedure. ${ }^{20,21}$ Therefore, in the appropriate patient population, and in the hands of an experienced operator, this technique may aid in assessment of femoral hernia reducibility. Radiologists and sonographers, however, may not possess sufficient experience in performing such a maneuver and may not be equipped to assess and treat the patient should worsening pain or other complication develop. Computed tomography will likely continue to play a large role in the diagnosis of emergent presentations of femoral hernias, given its rapid acquisition, ability to characterize the contents of the hernia sac, and capability of detecting signs and complications of bowel compromise. ${ }^{22}$

Figure 2. Images from a 21-year-old man with right groin pain. A, PreValsalva sonogram in the axial plane just caudad to the inguinal ligament shows the normal anatomic landmarks seen with the optimal technique, including the femoral artery $(A)$, femoral vein $(V)$, normal echogenic fat within the femoral canal $(F)$, pectineus muscle $(P)$, superior pubic ramus (arrows), and iliopsoas muscle (I). No hernia is present. Inset illustration shows the transducer position on the patient. B, Sonogram during the Valsalva maneuver in the same location shows normal inguinal anatomy and expected dilatation of the femoral vein. Note the femoral artery (A), femoral vein $(V)$, normal echogenic fat within the femoral canal $(F)$, pectineus muscle (P), superior pubic ramus (arrows), and iliopsoas muscle (I). C, Sonogram during the Valsalva maneuver shows normal inguinal anatomy in the oblique longitudinal plane. The transducer is held along the long axis of the femoral canal, parallel to the long axis of the femoral vessels, just medial to the femoral vein, showing the normal rounded caudal/distal contour of the peritoneal cavity (arrows). Note subcutaneous tissues (S) and difficult-to-visualize inguinal ligament (L).

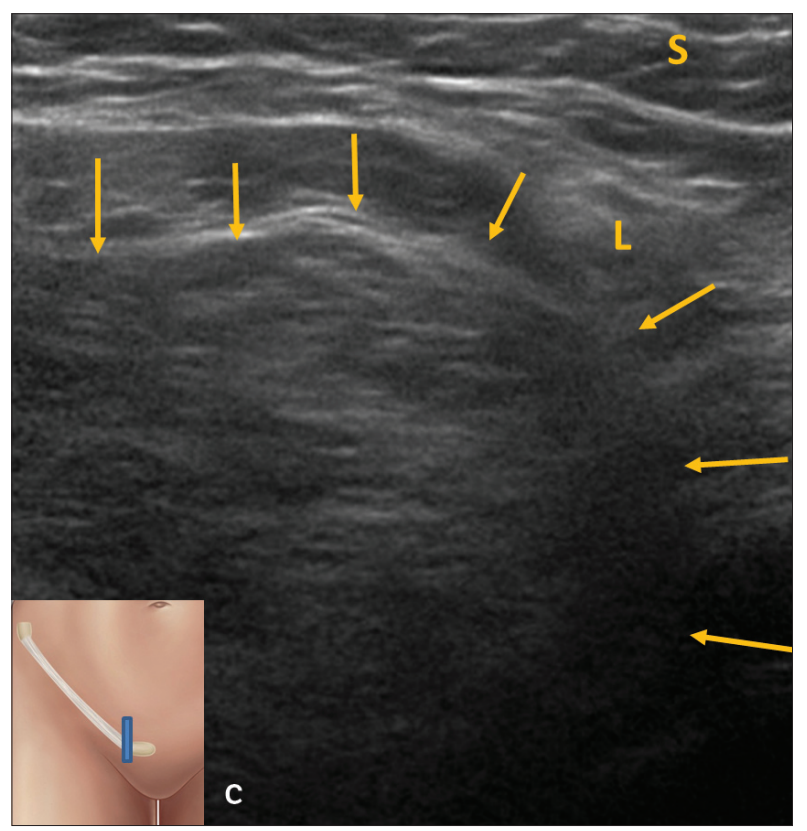


Our results also support the previous observations that although hernias are overall more likely to occur in men, women are relatively more likely to have femoral hernias. ${ }^{9,23}$ In a national review of surgically diagnosed hernias in adults without preoperative imaging, femoral hernias constituted $1.1 \%$ of groin hernias in men and $23 \%$ in women. ${ }^{24}$ In our small sample, $57 \%$ ( 8 of 14 ) of the hernias in women were femoral, compared to $17 \%$ ( 7 of 41 ) in men. The women in our population with a diagnosis of a femoral hernia also had higher mean and median ages. Forty percent (6 of 15) of our patients with surgically confirmed femoral hernias also had ipsilateral inguinal hernias diagnosed during surgical exploration. Although our methods differed
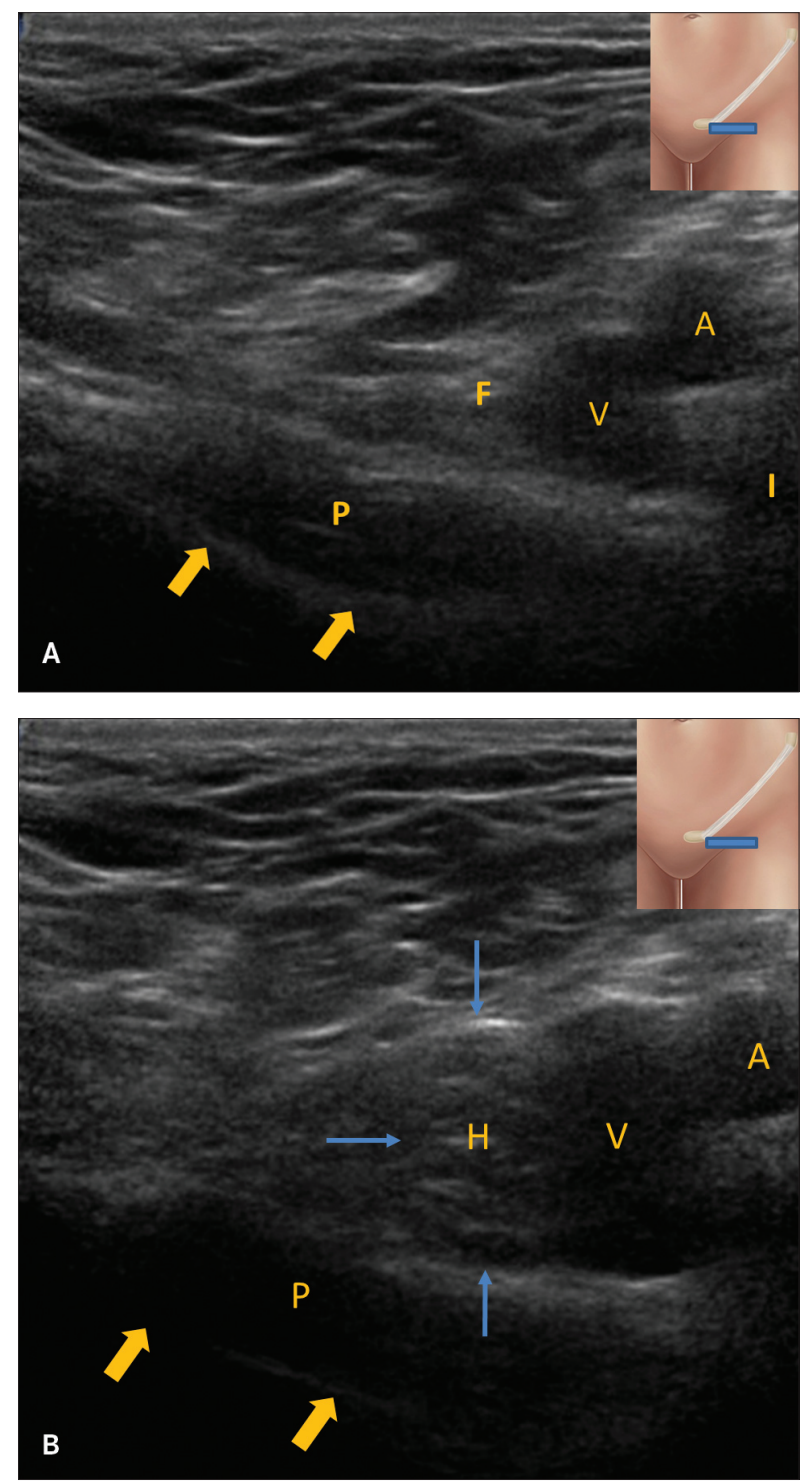

from examples in the surgical literature (our patients had physical examinations that were equivocal [occult], and the hernias were therefore diagnosed solely with imaging), our findings are concordant with several surgical series that found femoral hernias associated with simultaneous inguinal hernias in about $30 \%$ to $50 \%$ of patients. ${ }^{24,25}$ These authors also often found occult femoral hernias in reoperations for recurrent inguinal hernias; however, our study excluded patients who had undergone prior groin hernia repair, precluding our assessment of this finding.

In conclusion, despite the numerous advances in sonographic technology since the pioneer article published by Deitch and Soncrant ${ }^{13}$ in 1981, few additional scientific

Figure 3. Images from a 24-year-old man with a classic left femoral hernia. A, Pre-Valsalva sonogram in the axial plane just caudad to the inguinal ligament shows a normal appearance of the femoral artery $(A)$, femoral vein $(V)$, and echogenic fat within the femoral canal $(F)$. Note the superior pubic ramus (arrows), pectineus muscle (P), and iliopsoas muscle (I). The hernia is not shown in this image. B, Sonogram during the Valsalva maneuver in the same plane shows a hernia sac $(\mathrm{H}$, outlined with blue arrows) deforming the rounded medial contour of the femoral vein (V). Note the superior pubic ramus (yellow arrows), pectineus muscle (P), and femoral artery (A). C, Sonogram during the Valsalva maneuver in the longitudinal axis of the femoral canal just medial to the femoral vein shows a hernia sac (blue arrowheads) protruding beyond the normal rounded contour of the caudal peritoneal cavity (yellow arrows). See Figure $2 \mathrm{C}$ for normal longitudinal sonographic anatomy. Note the subcutaneous tissues (S) and inguinal ligament (L).

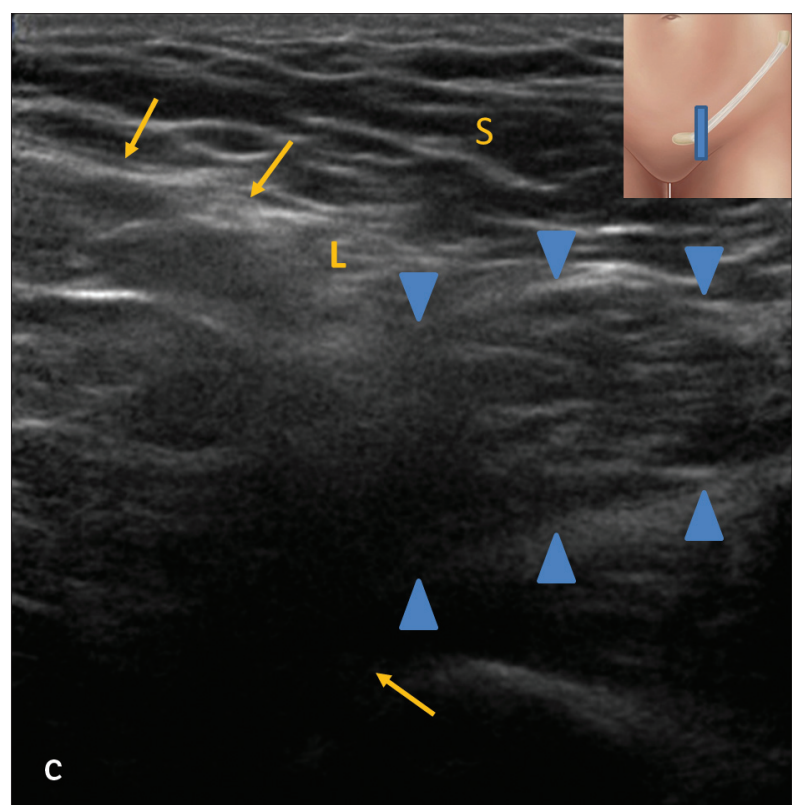


contributions regarding the utility of sonographic evaluation for assessment of occult femoral hernias exist. During our review, we made several observations regarding interpretation of sonographic findings. First, there is fat occupying the femoral canal during normal circumstances that is relatively echogenic. Second, a hernia sac contains relatively hypoechoic preperitoneal fat that displaces the normal more echogenic fat with increased intra-abdominal pressure. Third, a small amount of movement within the femoral canal can be normal, and to confidently make the diagnosis of a hernia, one should observe the hernia sac effacing the femoral vein and moving proximally to distally parallel to the femoral vessels.
We conclude that sonography is a useful clinical adjunct to physical examination, with good sensitivity and specificity and a high negative predictive value. Therefore, a clinician can reliably rule out a femoral hernia in the context of negative sonographic findings when following the proper techniques. Our results also suggest that with the proper technique, well-defined imaging criteria, and experience, the rate of false-positive diagnoses can be diminished. Larger prospective studies are indicated to further validate these results and may help determine the range of true positive predictive values of sonographic diagnosis of occult femoral hernias.

Table 1. Sonographic Diagnostic Considerations

Useful Characteristics of the Hernia Sac

Hypoechoic to normal femoral canal fat, with speckled internal echoes Increase in caliber of the femoral canal, with effacement of the femoral vein Movement caudad to the inguinal ligament in the longitudinal plane

\section{Potential Pitfalls}

Normal movement of femoral canal fat during Valsalva Normal enlargement of the vein during Valsalva Improper positioning, suboptimal Valsalva
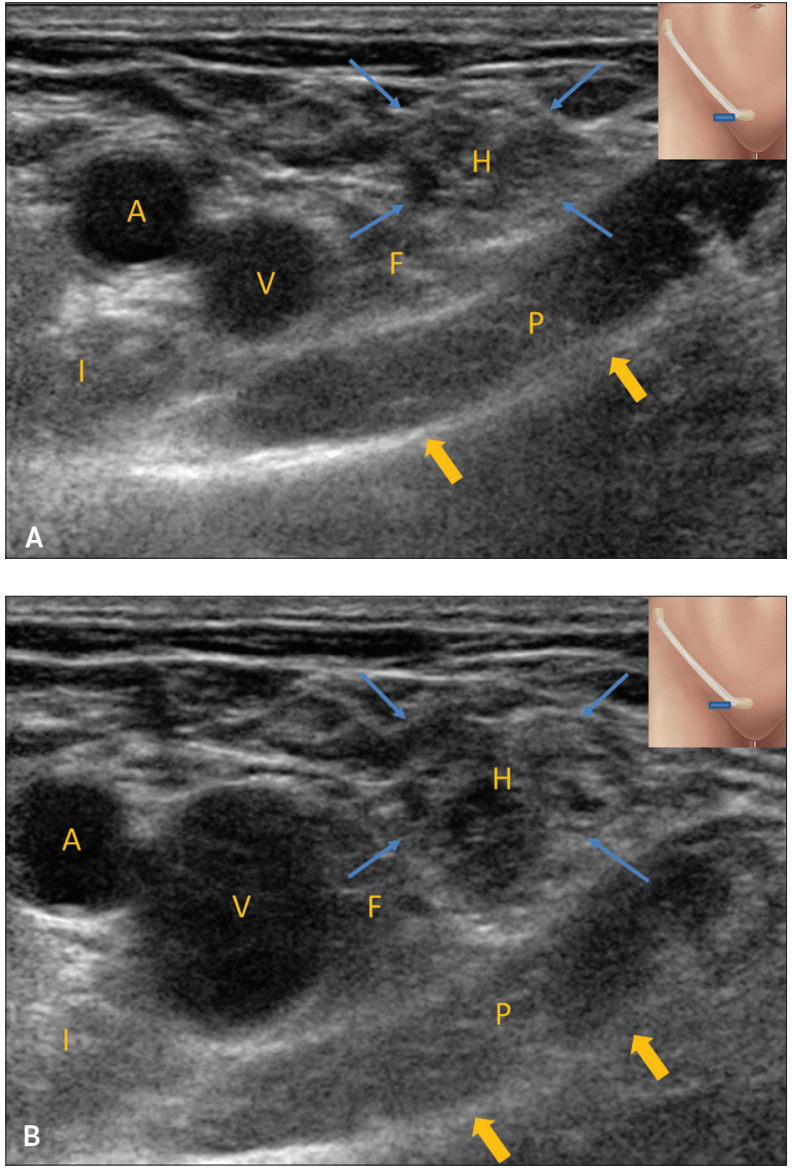

Figure 4. Images from a 36-year-old woman with an incarcerated right femoral hernia (uncommon medial variant). A, Pre-Valsalva sonogram in the axial plane just caudad to the inguinal ligament shows a hernia sac $(\mathrm{H}$, outlined with blue arrows) medial to the femoral canal $(F)$. There is no associated effacement of the femoral vein $(\mathrm{V})$. Note the femoral artery (A), iliopsoas muscle (I), pectineus muscle (P), and superior pubic ramus (yellow arrows). B, Sonogram during the Valsalva maneuver in the same axial plane shows mild bulging of the hernia sac $(\mathrm{H}$, outlined with blue arrows) but no demonstrable effacement of the femoral vein (V). Note the femoral artery (A), iliopsoas muscle (I), pectineus muscle (P), and superior pubic ramus (yellow arrows). F indicates femoral canal. C, Sonogram during the Valsalva maneuver in the longitudinal plane of the femoral vessels, $1 \mathrm{~cm}$ medial to the femoral vein, shows a hernia sac (blue arrowheads) extending beyond the normal rounded contour of the caudal peritoneal cavity (yellow arrows). Fluid ( $\mathrm{Fl}$ ) is shown along the distal aspect of the hernia sac, suggesting incarceration.).

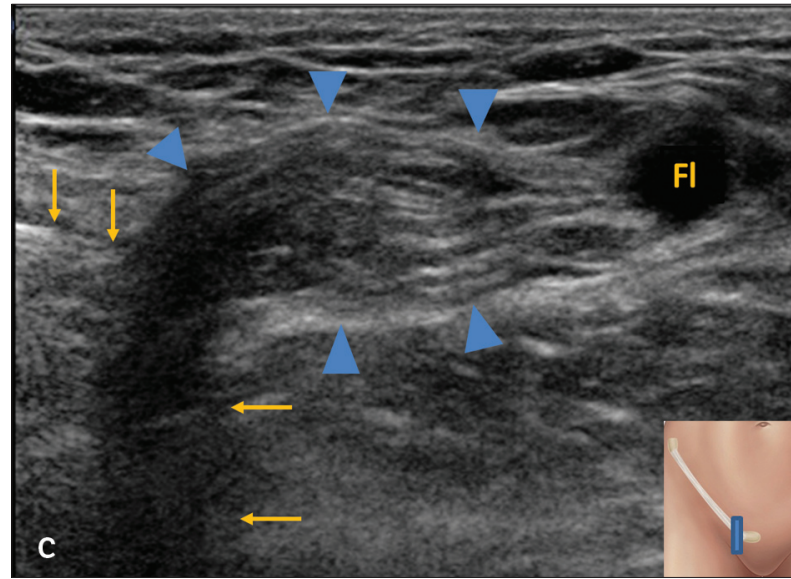




\section{References}

1. Rutkow IM. Demographic and socioeconomic aspects of hernia repair in the United States in 2003. Surg Clin North Am 2003; 83:1045-1051.

2. Kark A, Kurzer M, Waters KJ. Accuracy of clinical diagnosis of direct and indirect inguinal hernia. Br J Surg 1994; 81:1081-1082.

3. Robinson A, Light D, Nice C. Meta-analysis of sonography in the diagnosis of inguinal hernias. JUltrasound Med 2013; 32:339-346.

4. Jamadar DA, Jacobson JA, Morag Y, et al. Sonography of inguinal region hernias. AJR Am J Roentgenol 2006; 187:185-190.

5. Bradley M, Morgan D, Pentlow B, Roe A. The groin hernia: an ultrasound diagnosis? Ann R Coll Surg Engl 2003; 85:178-180.

6. Bradley M, Morgan J, Pentlow B, Roe A. The positive predictive value of diagnostic ultrasound for occult herniae. Ann R Coll Surg Engl 2006; 88:165-167.

7. Lilly MC, Arregui ME. Ultrasound of the inguinal floor for evaluation of hernias. Surg Endosc 2002; 16:659-662.

8. van den BergJC, de Valois JC, Go PM, Rosenbusch G. Detection of groin hernia with physical examination, ultrasound, and MRI compared with laparoscopic findings. Invest Radiol 1999; 34:739-743.

9. Humes DJ, Radcliffe RS, Camm C, West J. Population-based study of presentation and adverse outcomes after femoral hernia surgery. BrJ Surg 2013; 100:1827-1832.

10. Hachisuka T. Femoral hernia repair. Surg Clin North Am 2003; 83:11891205.

11. Naude GP, Ocon S, Bongard F. Femoral hernia: the dire consequences of a missed diagnosis. Am J Emerg Med 1997; 15:680-682.

12. Nilsson H, Stylianidis G, Haapamaki M, Nilsson E, Nordin P. Mortality after groin hernia surgery. Ann Surg 2007; 245:656-660.

13. Deitch EA, Soncrant MC. The value of ultrasound in the diagnosis of nonpalpable femoral hernias. Arch Surg 1981; 116:185-187.

14. Robinson P, Hensor E, Lansdown MJ, Ambrose NS, Chapman AH. Inguinofemoral hernia: accuracy of sonography in patients with indeterminate clinical features. AJR Am J Roentgenol 2006; 187:1168-1178.

15. Robinson A, Light D, Kasim A, Nice C. A systematic review and metaanalysis of the role of radiology in the diagnosis of occult inguinal hernia. Surg Endosc 2013; 27:11-18.

16. Depasquale R, Landes C, Doyle G. Audit of ultrasound and decision to operate in groin pain of unknown aetiology with ultrasound technique explained. Clin Radiol 2009; 64:608-614.

17. Rettenbacher $\mathrm{T}$, Hollerweger A, Macheiner P, et al. Abdominal wall hernias: cross-sectional imaging signs of incarceration determined with sonography. AJR Am J Roentgenol 2001; 177:1061-1066.

18. Loftus WK, Hewitt PM, Metreweli C. Case report: femoral hernia causing small bowel obstruction —ultrasound diagnosis. Clin Radiol 1998; 53:618-619.

19. Weng TI, Wang HP, Chen WJ, Chin LB, Ng LM. Ultrasound diagnosis of occult femoral hernia presenting with intestinal obstruction. Am JEmerg Med 2001; 19:333-334.

20. Chen S, Lee C, Liu Y, et al. Ultrasound may decrease the emergency surgery rate of incarcerated inguinal hernia. Scand J Gatroenterol 2005; 40:721-724.

21. Siadecki SD, Frasure SE, Saul T, Lewiss RE. Diagnosis and reduction of a hernia by bedside ultrasound: a case report. J Emerg Med 2014; 47:169 171.

22. Aguirre DA, Casola G, Sirlin C. Abdominal wall hernias: MDCT findings. AJR Am J Roentgenol 2004; 183:681-690.

23. Dahlstrand U, Wollert S, Nordin P, Sandblom G, Gunnarsson U. Emergency femoral hernia repair: a study based on a national register. Ann Surg 2009; 249:672-676.

24. Chan G, Chan CK. Long term results of a prospective study of 225 femoral hernia repairs: indications for tissue and mesh repair. J Am Coll Surg 2008; 207:360-367.

25. Koch A, Edwards A, Haapaniemi S, Nordin P, Kald A. Prospective evaluation of 6895 groin hernia repairs in women. Br J Surg 2005; 92:15531558. 